\title{
Combined Analyses of hENT1, TS, and DPD Predict Outcomes of Borderline-resectable Pancreatic Cancer
}

\author{
YASUHIRO YABUSHITA ${ }^{1}$, RYUTARO MORI ${ }^{1}$, KOICHI TANIGUCHI $^{1}$, RYUSEI MATSUYAMA $^{1}$, \\ TAKAFUMI KUMAMOTO ${ }^{1}$, KENTARO SAKAMAKI ${ }^{2}$, KENSUKE KUBOTA $^{3}$ and ITARU ENDO ${ }^{1}$ \\ ${ }^{1}$ Department of Gastroenterological Surgery, Yokohama City University School of Medicine, Yokohama, Japan; \\ ${ }^{2}$ Department of Biostatistics, Yokohama City University Medical Center, Yokohama, Japan; \\ ${ }^{3}$ Department of Gastroenterology, Yokohama City University School of Medicine, Yokohama, Japan
}

\begin{abstract}
Background: Predicting chemosensitivity to neoadjuvant chemoradiotherapy (NACRT) in pancreatic cancer is desired. The present study aimed to examine the relationship between intratumoral expression of human equilibrative nucleoside transporter 1 (hENT1), thymidylate synthase (TS), and dihydropyrimidine dehydrogenase (DPD) and the outcomes of NACRT with gemcitabine (GEM) combined with $S-1$ in patients with borderline-resectable pancreatic cancer (BRPC). Materials and Methods: Forty-seven patients who underwent NACRT with GEM plus S-1, following curative surgery, were recruited in our Institution between 2009 and 2012. Immunohistochemical expressions of hENT1, TS, and DPD in fine-needle aspiration (FNA) biopsies and resected specimens were examined. The correlation between these enzyme expressions and long-term outcome was analyzed. Results: In 21 FNA specimens, no relationship between clinical responses to NACRT and long-term survival was found. However, in 47 resected specimens, patients were classified according to the number of favorable hENT1, TS, and DPD expression factors (hENTI positive/TS negative/DPD negative). The presence of three favorable factors was strongly associated with improved partial response rates to NACRT $(p=0.002)$. Patients with 2 or more favorable factors showed a significantly longer overall survival than the other patients $(p=0.002)$. Conclusion: Combined expression analyses of hENT1, TS, and DPD may predict long-term outcomes in patients with $B R P C$ after NACRT.
\end{abstract}

Correspondence to: Itaru Endo, MD, Ph.D, Department of Gastroenterological Surgery, Yokohama City University School of Medicine, Yokohama, Japan. Tel: +81 457872650, Fax: +81 457829161, e-mail: endoit@yokohama-cu.ac.jp

Key Words: hENT1, TS, DPD, borderline-resectable pancreatic cancer, NACRT.
Pancreatic cancer is a highly lethal disease (1). Complete surgical resection of tumors remains the only treatment with curative potential. However, up to $20 \%$ of patients are eligible for surgical resection at diagnosis (2). Furthermore, surgical resection cannot guarantee a cure, although the 5year survival rate improves to approximately $10 \%$ following resection (3). Several randomized controlled studies of adjuvant chemotherapy after pancreatic resection have demonstrated survival benefits (4-6). According to these results, adjuvant chemotherapy is currently considered to be a standard treatment and is routinely recommended.

Evans et al. (7) first reported the use of neoadjuvant chemoradiotherapy (NACRT) for pancreatic cancer to improve the R0 resection and patient survival rates. In 2008, Katz et al. (8) proposed the concept of borderline-resectable pancreatic cancer (BRPC), which is at a high risk for a margin-positive resection with surgery alone. In practice, a Japanese multicenter study found that the R0 resection rate for patients with BRPC was $65.9 \%$ and that the 3- and 5-year survival rates in resected patients were $22.8 \%$ and $12.5 \%$, respectively (9). In this study, only $9.4 \%$ of patients underwent preoperative adjuvant treatment. To increase the R0 resection and overall survival (OS) rates, NACRT has been introduced for BRPC. Moreover, in 2009, our department adopted neoadjuvant gemcitabine (GEM) plus S1 combination chemoradiotherapy.

Chemotherapy with GEM (2',2'-difluirideoxycytidine), a deoxycytidine analog that inhibits DNA replication and repair, is one of the standard treatment for pancreatic cancer. The human equilibrative nucleotide transporter 1 (hENT1) protein plays an important role for transporting GEM to intracellular space (10) and can also predict chemosensitivity for GEM-based treatment. Less hENT1 expression in cancer cells induces lower intracellular concentration of GEM, resulting in drug resistance (11). Our previous reports revealed that human pancreatic cancer cells with $h E N T 1$ expression are shown to be sensitive to GEM (12). Furthermore, a randomized study demonstrated that hENT1 
protein expression was associated with prolonged OS and disease-free survival (DFS) rates in patients who received postoperative GEM (13). Therefore, hENT1 expression may be a predictive factor of the survival rate in patients with pancreatic cancer undergoing GEM chemotherapy.

S-1, an oral fluoropyrimidine derivative comprising of tegafur and the two modulators 5-chloro-2,4-dihydroxypyridine and potassium oxonate, has been used to treat patients with pancreatic cancer since the early 2000s in Japan. The GEST study, a multicenter, prospective, randomized study for unresectable pancreatic cancer, demonstrated that S-1 and GEM had similar efficacies in the disease (14). Furthermore, GEM plus S-1 did not demonstrate superiority with respect to OS. However, the response rate for GEM plus S-1 was $29.3 \%$, which is higher than that for GEM or S-1 alone. Hence, assessing pancreatic tumor cell sensitivity to 5-fluorouracil (5-FU) is important and is usually performed by measuring the expression levels of thymidylate synthase (TS) and dihydropyrimidine dehydrogenase (DPD) in cancer cells $(15,16)$. Studies have evaluated the correlation between TS and DPD expression levels in tumors and clinical outcomes for patients with gastric cancer $(17,18)$.

To evaluate the prognostic value of hENT1, TS, and DPD expression for long-term outcomes of patients with BRPC undergoing NACRT with GEM plus S-1, we assessed the protein expression levels of these enzymes in tumor samples using fine-needle aspiration (FNA) and resected specimens and identified the correlations with clinical parameters and disease outcomes.

\section{Patients and Methods}

Patients. Fifty-seven patients with pancreatic cancer visited the Department of Gastroenterological Surgery, Yokohama City University Hospital between January 2009 and December 2012 and were enrolled in our study. All patients gave their informed consent, and the study was conducted according to the Declaration of Helsinki. The Ethics Committee of the hospital approved the present study (No. B090312028). Diagnoses of pancreatic cancer were confirmed by cytology in 27 patients and by histology of specimens using endoscopic ultrasonography-guided FNA (EUS-FNA) biopsies for 30 patients.

In this study, the indication for NACRT was as follows: patients with BRPC, defined by the National Comprehensive Cancer Network Guidelines Version 1.2013 (19), and with Eastern Cooperative Oncology Group performance status 0-1. On completing chemoradiotherapy, restaging $\mathrm{CT}$ and positron emission tomography were performed. If distant metastasis or local progression were not present, surgery was performed.

Treatment planning and assessments of clinical efficacy. NACRT comprised of combined chemotherapy of GEM and S-1 followed by radiotherapy. GEM plus S-1 comprised of the intravenous administration of $1,000 \mathrm{mg} / \mathrm{m}^{2} \mathrm{GEM}$ on days 8 and 15 and daily oral administration of $60 \mathrm{mg} / \mathrm{m}^{2} \mathrm{~S}-1$ on days $1-14$. After two courses of the chemotherapy, radiotherapy (30 Gy) was combined with $60 \mathrm{mg} / \mathrm{m}^{2} \mathrm{~S}-1$. Patients received restaging interventions at 4-6 weeks after completing chemoradiotherapy. If there was no disease progression, the patients underwent surgery. Clinical responses to NACRT were assessed using the Response Evaluation Criteria in Solid Tumors (RECIST) criteria (20).

Resection procedures and postoperative chemotherapy. Ten patients were excluded from this study: six had liver metastases and four had peritoneal dissemination after chemoradiotherapy. Thus, 47 (82\%) patients underwent surgical resection after NACRT. After surgery, postoperative chemotherapy was planned, comprising of a regimen of intravenously administered 1,000 $\mathrm{mg} / \mathrm{m}^{2}$ GEM on days 8 and 15 plus orally-administered $60 \mathrm{mg} / \mathrm{m}^{2}$ S-1 on days 1-14 for 6 months. After surgical resection, all the patients were followed up monthly, and serum carcinoembryonic antigenand CA19-9 levels were determined every 2 or 3 months. MDCT was performed every 3 months for 2 years and every 6 months thereafter. Patients with elevated tumor markers were immediately examined by MDCT. When recurrence ocurred, the recurrent site was recorded.

Initial histopathological assessment. Sections were routinely histologically examined. Residual tumors were considered as R1 if histological infiltration of carcinoma was present at the pancreatic cut end margin, at the bile duct cut end margin, or in the dissected peripancreatic tissue margin. Tumor stage, lymph node metastasis, and final stage were classified according to the 7th edition of the International Union Against Cancer (UICC) tumor, node, and metastasis system (TNM) system (21). Histological responses to NACRT were determined following Evans classification (3); grade 1, slight tumor cell destruction $(<10$ $\%$ ); grade $2 \mathrm{a}, 10 \%-50 \%$ non-viable tumor cells; grade $2 \mathrm{~b}, 50-90 \%$ non-viable tumor cells; grade $3, \leq 10 \%$ viable tumor cells; and grade 4 , no viable tumor cells.

Immunohistochemical staining. Tissue sections of $4 \mu \mathrm{m}$ were deparaffinized, and endogenous peroxidase activity of specimens was blocked by immersing the slides in a solution of absolute methanol containing $0.3 \%$ hydrogen peroxide for $30 \mathrm{~min}$ at room temperature. To retrieve antigens, slides were autoclaved at $105^{\circ} \mathrm{C}$ for $10 \mathrm{~min}$ in the Dako Target Retrieval Solution High $\mathrm{pH} \times 1$ for hENT 1 or at $121^{\circ} \mathrm{C}$ for $10 \mathrm{~min}$ in $1 \mathrm{M}$ methylenediamine tetraacetic acid buffer for DPD and TS. After autoclaving, sections were put at room temperature for cooling. After blocking endogenous peroxidases, tissue slices were incubated with 1:100 dilutions of anti-hENT1 rabbit polyclonal antibody (Abnova, Taipei, Taiwan), 1:50 dilutions for anti-DPD mouse monoclonal antibody (IBL, Gunma, Japan), or 1:200 dilutions for anti-TS mouse monoclonal antibody (IBL, Gunma, Japan) at $4{ }^{\circ} \mathrm{C}$ overnight. Labeled antigens were visualized using a Histo Fine kit (Nichirei, Tokyo, Japan), followed by reaction with DAB (Wako, Osaka, Japan). Finally, sections were counterstained with hematoxylin and observed under a microscope. Negative control was prepared in the absence of primary antibodies.

Immunohistochemical evaluation. Expression levels of hENT1, TS, and DPD were evaluated in 21 FNA specimens and 47 resected specimens by immunohistochemistry. Staining grades were evaluated by two observers (Y.Y. and R.M.) under the direction of 

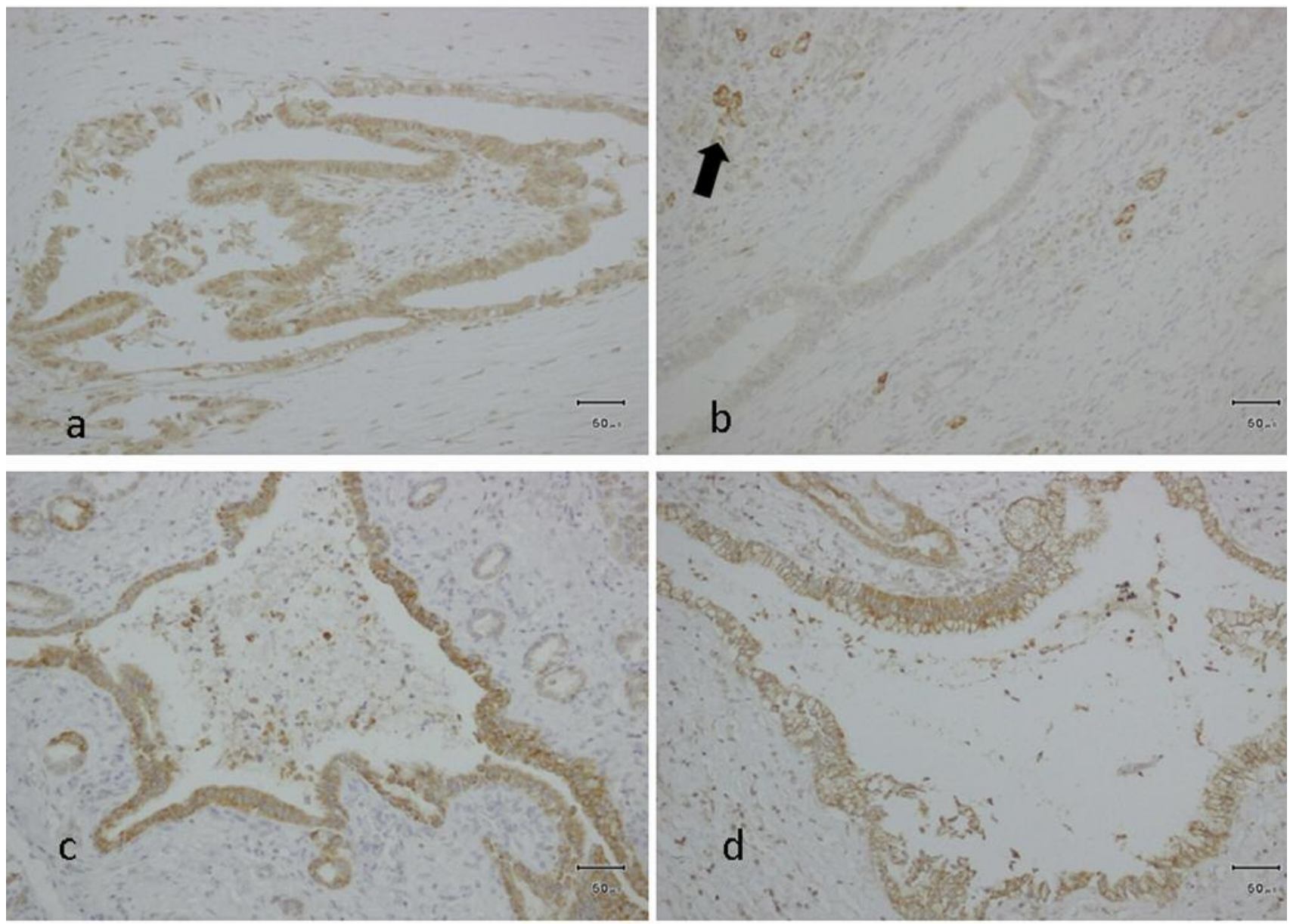

Figure 1. Immunohistochemical analysis of hENT1, TS, and DPD expression in pancreatic cancer. (a) Positive hENT1 expression. (b) Negative hENT1 expression stained adjacent islet cells (arrow) were used as a positive internal control. (c) Positive TS expression. (d) Positive DPD expression. Scale bar, $50 \mu \mathrm{m}$.

a pathologist. Clinical information was blinded at the time of evaluation. Islets of Langerhans cells were selected as the internal positive control for hENT1 and DPD expression. The intensity of hENT1 and DPD staining was scored as follows: grade 0 , no staining; grade 1 , faintly stained; grade 2 , weakly stained compared with islet cells; and grade 3, stained as strongly as islet cells. TS expression level was quantified using a visual grading system on the basis of the extent of staining. The intensity of TS staining was scored from grades 0 to 3 as previously reported (grade 0 , no staining; grade 1, light yellow; grade 2, yellow or deep yellow; and grade 3 , brown or tan) (22). Samples were considered hENT1 positive if grade 2 or 3 staining was observed in $>50 \%$ of carcinoma cells. Samples were considered TS- or DPD-positive if grade 2 or 3 staining with $30 \%$ or more of carcinoma cells. These cutoff values were decided according to previous reports $(13,23)$. Samples that did not fulfill these criteria were determined as negative for the expression of tumor markers (Figure 1).

Statistical analyses. Statistical analyses were performed using the SPSS version 20 software (SPSS Inc., Chicago, IL, USA). Observation period and patients' age were presented as median and range. Comparisons of categorical variables were tested by $\chi^{2}$ or Fisher's exact tests. Significant differences within groups were identified using paired $t$-tests and across groups using the MannWhitney $U$-test. DFS and OS curves were estimated using KaplanMeier analyses, and differences between patient groups were evaluated using the log-rank test. Multivariate Cox proportional hazard analyses were performed to determine the influences of variables on outcomes using a stepwise variable selection process. Differences were considered statistically significant at $\mathrm{p}<0.05$.

\section{Results}

Patient population and clinical outcomes. Patient characteristics are summarized in Table I. Thirty-one $(66 \%)$ patients were males and $16(34 \%)$ were females, with a median age of 65 (37-81) years. Pancreatic tumors were confined to the head and body-tail of the pancreas in $36(77 \%)$ and 11 (33\%) patients, respectively. Forty-six patients completed the two planned courses of chemotherapy. Because of an allergic reaction, one patient underwent NACRT without oral 
Table I. Characteristics of the 47 patients.

\begin{tabular}{lc}
\hline Characteristics & $\mathrm{N}=47$ \\
\hline Age & \\
$\quad<65$ & 23 \\
$\geq 65$ & 24 \\
Gender & \\
Male & 31 \\
Female & 16 \\
Tumor location & \\
Head & 36 \\
Body-Tail & 11 \\
UICC T classification & \\
T3 & 31 \\
T4 & 16 \\
Operative procedure & \\
Pancreaticoduodenectomy & 36 \\
Distal pancreatectomy & 11 \\
Total pancreatectomy & 0 \\
Major vessel resection & \\
Yes & 37 \\
PV/SMV & 29 \\
SMA & 2 \\
CHA & 2 \\
CA & 8 \\
No & 10 \\
Surgical margin & \\
R0 & \\
R1 & \\
Pathological differentiation & \\
Well & \\
Others & \\
Lymph node metastasis & \\
Negative & \\
Positive & \\
\hline & \\
& \\
\hline &
\end{tabular}

administration of S-1. Twenty-one patients provided sufficient volumes of specimen for FNA analyses. The average number of FNA specimens from each patient was four (range=2-6). Resection procedures included pancreaticoduodenectomy in 36 (77\%) patients and distal pancreatectomy in 11 (33\%). Combined resection of major vessels was performed in 37 patients: PV/SMV in 29, SMA in two, common hepatic artery in two, and celiac artery in eight. The surgical margin was assessed as negative for malignancy (R0) in 43 (91\%) patients and microscopically positive (R1) in four (9\%). Tumors were identified as well-differentiated adenocarcinomas in seven (15\%) patients, moderately differentiated in 29 (62\%), poorly differentiated in $10(21 \%)$, and adenosquamous carcinoma in one (2\%). According to the TNM classification, $31(66 \%)$ and $16(34 \%)$ patients had T3 and T4 tumors, respectively, and 23 (49\%) patients had lymph node metastases. According to the RECIST criteria, NACRT produced partial response (PR) in 11 (23\%) patients, but stable disease in 36 (77\%). Pathological assessments using Evans criteria classified histological responses as grade 1 in two (4\%) patients, grade $2 \mathrm{a}$ in 22 $(47 \%)$, grade $2 \mathrm{~b}$ in $18(38 \%)$, and grade 3 in five $(11 \%)$. Twenty-seven (57\%) patients underwent postoperative chemotherapy with GEM plus S-1. Treatment was completed in 6 months for $14(52 \%)$ patients. The other 13 patients changed chemotherapy because of disease recurrence $(n=7$, $26 \%)$ and side effects $(n=6,22 \%)$. Four $(9 \%)$ patients received GEM alone, four (9\%) received S-1 alone, and 12 (26\%) did not received postoperative chemotherapy as per the patients' decision.

Median follow-up was 20 (6.5-51.9) months after the initial treatment. Median duration of DFS and OS after initial treatment were 15.4 and 24.8 months, respectively, and 1and 3-year DFS and OS rates of the entire cohort were $62.2 \%$ and $32.2 \%$ and $91.1 \%$ and $34.0 \%$, respectively.

Clinicopathological factors and hENT1, TS, and DPD expression. Among 21 FNA specimens, seven (33\%) were hENT1 positive, five (24\%) were TS positive, and $13(62 \%)$ were DPD positive. Among eight clinicopathological factors (age, sex, tumor location, UICC classification, major vessel resection, surgical margin, pathological differentiation, and lymph node metastasis), no significant differences in characteristics were observed between the positive and negative groups (Table II). Relationships between hENT1, TS, and DPD expression levels in FNA and in resected specimens are presented in Table III. No correlations between expression levels in FNA and resected specimens were found.

Among 47 resected tumors, 26 (55\%) were hENT1 positive, whereas $26(55 \%)$ were TS positive and $32(68 \%)$ were DPD positive. However, no significant differences were observed in eight clinicopathological factors between these marker expression groups (Table IV).

Patients were classified into one of the following four groups according to the number of favorable hENT1, TS, and DPD expression factors: three favorable factors (hENT1 positive/TS negative/DPD negative; $n=6$ ), any two favorable factors (hENT1 positive/TS negative/DPD positive, hENT1 positive/TS positive/DPD negative, or hENT1 negative/TS negative/DPD negative; $n=13$ ), any one favorable factor ( $h E N T 1$ positive/TS positive/DPD positive, hENT1 negative/ TS negative/DPD positive, or hENT1 negative/TS positive/DPD negative; $n=21$ ), or no favorable factors (hENT1 negative/TS positive/DPD positive; $n=7)$. Forty $(85 \%)$ patients revealed the presence of more than one favorable factor, $19(40 \%)$ the presence of more than two, and six (13\%) the presence and absence of three favorable factors. The tumor location of patients with three favorable factors was significantly more frequent in the bodytail of the pancreas (Table V).

Relationships between tumor response to NACRT and hENT1, $T S$, and DPD expression and their combination. Relationships between hENT1, TS, and DPD expression and tumor responses 
Table II. Comparison of clinicopathological factors based on intratumoral hENT1, TS, and DPD expression in FNA specimens ( $n=21$ ).

\begin{tabular}{|c|c|c|c|c|c|c|c|c|c|}
\hline & \multicolumn{2}{|c|}{ hENT1 } & \multirow[t]{2}{*}{$p$-Value } & \multicolumn{2}{|c|}{ TS } & \multirow[t]{2}{*}{$p$-Value } & \multicolumn{2}{|c|}{ DPD } & \multirow[t]{2}{*}{$p$-Value } \\
\hline & $\begin{array}{l}\text { Positive } \\
(\mathrm{n}=7)\end{array}$ & $\begin{array}{c}\text { Negative } \\
(\mathrm{n}=14)\end{array}$ & & $\begin{array}{l}\text { Positive } \\
(\mathrm{n}=5)\end{array}$ & $\begin{array}{c}\text { Negative } \\
(\mathrm{n}=16)\end{array}$ & & $\begin{array}{c}\text { Positive } \\
(\mathrm{n}=13)\end{array}$ & $\begin{array}{c}\text { Negative } \\
\quad(\mathrm{n}=8)\end{array}$ & \\
\hline Age & & & 0.438 & & & 0.185 & & & 0.392 \\
\hline$<65$ & 4 & 6 & & 1 & 9 & & 7 & 3 & \\
\hline$\geq 65$ & 3 & 8 & & 4 & 7 & & 6 & 5 & \\
\hline Gender & & & 0.557 & & & 0.656 & & & 0.310 \\
\hline Male & 4 & 9 & & 2 & 6 & & 6 & 2 & \\
\hline Female & 3 & 5 & & 3 & 10 & & 7 & 6 & \\
\hline Tumor location & & & 0.681 & & & 0.258 & & & 0.160 \\
\hline Head & 4 & 8 & & 4 & 8 & & 9 & 3 & \\
\hline Body-Tail & 3 & 6 & & 1 & 8 & & 4 & 5 & \\
\hline UICC $\mathrm{T}$ classification & & & 0.676 & & & 0.080 & & & 0.528 \\
\hline T3 & 3 & 6 & & 4 & 5 & & 6 & 3 & \\
\hline $\mathrm{T} 4$ & 4 & 8 & & 1 & 11 & & 7 & 5 & \\
\hline Major vessel resection & & & 0.274 & & & 0.579 & & & 0.316 \\
\hline Yes & 7 & 11 & & 4 & 14 & & 12 & 6 & \\
\hline No & 0 & 3 & & 1 & 2 & & 1 & 2 & \\
\hline Surgical margin & & & 0.667 & & & 0.381 & & & 0.381 \\
\hline R0 & 7 & 13 & & 5 & 15 & & 13 & 7 & \\
\hline $\mathrm{R} 1$ & 0 & 1 & & 0 & 1 & & 0 & 1 & \\
\hline Pathological differentiation & & & 0.433 & & & 0.571 & & & 0.133 \\
\hline Well & 0 & 2 & & 0 & 2 & & 0 & 2 & \\
\hline Others & 7 & 12 & & 5 & 14 & & 13 & 6 & \\
\hline Lymph node metastasis & & & 0.056 & & & 0.262 & & & 0.664 \\
\hline Negative & 2 & 11 & & 2 & 11 & & 8 & 5 & \\
\hline Positive & 5 & 3 & & 3 & 5 & & 5 & 3 & \\
\hline
\end{tabular}

to NACRT were evaluated. Clinical efficacy, including radiographic and histological responses, did not significantly differ between patients with marker-positive and -negative FNA specimens. In contrast, resected specimens that were DPD negative tended to demonstrate treatment effects in radiographic assessments $(p=0.073)$. Evans' histopathological criteria did not significantly differ between patients grouped according to marker expression (Table VI). The PR rates of NACRT were strongly associated with the presence of all three favorable factors $(p=0.002)$. Furthermore, any $\geq 2$ favorable factors $(\mathrm{n}=19,40 \%)$ tended to demonstrate PR $(p=0.074)$.

Relationship between survival and hENT1, TS, and DPD expression. No relationships were found between expressions of marker proteins in FNA specimens and survival. However, patients with three favorable factors in resected specimens had significantly longer OS $(p=0.005)$ than patients in the other groups (Figure 2). Patients with more than two favorable factors tended to have longer DFS $(p=0.056)$ and OS $(p=0.002)$ rates than patients in the other groups (Figure 2).

Univariate analyses of DFS and OS are presented in Table VII. Patients with tumor in the body-tail had a significantly longer OS rate $(p=0.028)$. One- and 3 -year OS rates were
Table III. Correlation between the expression in FNA specimens and resected specimens.

\begin{tabular}{lrccr}
\hline & \multicolumn{3}{c}{ Resected specimens } & \\
\cline { 3 - 4 } & $\mathrm{N}$ & Positive (\%) & Negative(\%) & $p$-Value \\
\hline FNA-hENT & & & & 0.572 \\
$\quad$ Positive & 7 & $5(71)$ & $2(29)$ & \\
Negative & 14 & $9(64)$ & $5(36)$ & \\
FNA-TS & & & & 0.258 \\
Positive & 5 & $4(80)$ & $1(20)$ & \\
Negative & 16 & $8(50)$ & $8(50)$ & 0.590 \\
FNA-DPD & & & & \\
Positive & 13 & $9(69)$ & $4(31)$ & \\
Negative & 8 & $6(75)$ & $2(25)$ & \\
\hline
\end{tabular}

significantly higher in patients with TS-negative resected specimens (90\% and $65 \%$, respectively) than in those with TS-positive resected specimens (92\% and $10 \%$, respectively, $p=0.013)$. The DPD-negative group achieved significantly longer OS rate both at 1-year and 3-year (85\% and 59\%, respectively) than the DPD-positive group (94\% and $22 \%$, 
Table IV. Comparison of clinicopathological factors based on intratumoral hENT1, TS, and DPD expression in resected specimens ( $n=47$ ).

\begin{tabular}{|c|c|c|c|c|c|c|c|c|c|}
\hline & \multicolumn{2}{|c|}{ hENT1 } & \multirow[t]{2}{*}{$p$-Value } & \multicolumn{2}{|c|}{ TS } & \multirow[t]{2}{*}{$p$-Value } & \multicolumn{2}{|c|}{ DPD } & \multirow[t]{2}{*}{$p$-Value } \\
\hline & $\begin{array}{c}\text { Positive } \\
(n=26)\end{array}$ & $\begin{array}{c}\text { Negative } \\
(\mathrm{n}=21)\end{array}$ & & $\begin{array}{c}\text { Positive } \\
(n=26)\end{array}$ & $\begin{array}{c}\text { Negative } \\
(\mathrm{n}=21)\end{array}$ & & $\begin{array}{c}\text { Positive } \\
(\mathrm{n}=32)\end{array}$ & $\begin{array}{l}\text { Negative } \\
(\mathrm{n}=15)\end{array}$ & \\
\hline Age & & & 0.454 & & & 0.454 & & & 0.299 \\
\hline$<65$ & 14 & 9 & & 14 & 9 & & 14 & 9 & \\
\hline$\geq 65$ & 12 & 12 & & 12 & 12 & & 18 & 6 & \\
\hline Gender & & & 0.477 & & & 0.252 & & & 0.555 \\
\hline Male & 16 & 15 & & 19 & 12 & & 22 & 9 & \\
\hline Female & 10 & 6 & & 7 & 9 & & 10 & 6 & \\
\hline Tumor location & & & 0.390 & & & 0.610 & & & 0.229 \\
\hline Head & 19 & 17 & & 20 & 16 & & 26 & 10 & \\
\hline Body - Tail & 7 & 4 & & 6 & 5 & & 6 & 5 & \\
\hline UICC T classification & & & 0.477 & & & 0.927 & & & 0.944 \\
\hline $\mathrm{T} 3$ & 16 & 15 & & 17 & 14 & & 21 & 10 & \\
\hline $\mathrm{T} 4$ & 10 & 6 & & 9 & 7 & & 11 & 5 & \\
\hline Major vessel resection & & & 0.512 & & & 0.488 & & & 0.397 \\
\hline Yes & 20 & 17 & & 21 & 16 & & 26 & 11 & \\
\hline No & 6 & 4 & & 5 & 5 & & 6 & 4 & \\
\hline Surgical margin & & & 0.610 & & & 0.227 & & & 0.619 \\
\hline R0 & 24 & 19 & & 25 & 18 & & 29 & 14 & \\
\hline $\mathrm{R} 1$ & 2 & 2 & & 1 & 3 & & 3 & 1 & \\
\hline Pathological differentiation & & & 0.377 & & & 0.307 & & & 0.394 \\
\hline Well & 3 & 4 & & 5 & 2 & & 4 & 3 & \\
\hline Others & 23 & 17 & & 21 & 19 & & 28 & 12 & \\
\hline Lymph node metastasis & & & 0.312 & & & 0.454 & & & 0.401 \\
\hline Negative & 15 & 9 & & 12 & 12 & & 15 & 9 & \\
\hline Positive & 11 & 12 & & 14 & 9 & & 17 & 6 & \\
\hline
\end{tabular}

respectively, $p=0.035)$. However, no relationship was found between hENT1 expression and survival.

Multivariate Cox proportional models were fitted using prognostic factors which were significant in the univariate analysis. TS and DPD expressions were omitted from this analysis because "more than two favorable factors" included these factors. "Three favorable factors" was not added to this analysis because the number of patients was small. The two prognostic factors tumor location and more than two favorable factors were included in multivariate analyses of OS. These analyses revealed that the presence of more than two favorable factors was an independent factor influencing long-term survival rate (hazard ratio, 3.40; 95\% confidence interval, 1.36-8.68; $p=0.010$; Table VI).

\section{Discussion}

This study evaluated the influence of hENT1, TS, and DPD expression on NACRT with GEM and S-1 based regimen for patients with BRPC. A relationship between more than two favorable factors of three enzymes and survival benefit was observed. However, the 3-year OS rate of patients with an absence of more than two favorable factors was only $10 \%$.
Recently, the OS rates in patients with pancreatic cancer undergoing surgical resections following neoadjuvant therapy have increased. Since the clinical introduction of GEM (24) in the late 1990s, numerous randomized studies have suggested that GEM supersedes 5-FU which was the standard chemotherapeutic agent for pancreatic cancer. Although various studies indicated that efficacy of GEM was superior to 5-FU, 5-FU has been clearly demonstrated as an effective drug in patients with pancreatic cancer (5). Trials of adjuvant treatments, including the RTOG 9704 study (25), which was a large prospective randomized trial, and the CONKO-001 study (26), provided strong supporting evidence for the efficacy of adjuvant therapy. In particular, Evans et al. (27) reported an actual 5-year OS rate of $36 \%$ in patients with resectable pancreatic head cancer who underwent surgical resection following preoperative GEMbased chemoradiotherapy. Moreover, Motoi et al. (28) reported a median OS of 35 months in patients who received resection following neoadjuvant therapy with GEM plus S-1. Thus, GEM and fluoropyrimidine are pivotal agents in current adjuvant and neoadjuvant therapies. A Japanese multicenter study (9) found that the 3- and 5-year OS rates in patients with borderlineresected pancreatic cancer were 22.8 and $12.5 \%$, respectively. The present study shows a 3 -year OS rate of $34 \%$ and a median 
Table V. Comparison of clinicopathological factors based on intratumoral combined expression of hENT1-positive, T-negative, and DPD-negative in resected specimens. $(n=47)$

\begin{tabular}{|c|c|c|c|c|c|c|c|c|c|}
\hline & \multicolumn{2}{|c|}{ hENT1/TS/DPD } & \multirow[t]{2}{*}{$p$-Value } & \multicolumn{2}{|c|}{ hENT1/TS/DPD } & \multirow[t]{2}{*}{$p$-Value } & \multicolumn{2}{|c|}{ hENT1/TS/DPD } & \multirow[t]{2}{*}{$p$-Value } \\
\hline & $\begin{array}{c}\geq 1 \\
\text { matched } \\
(n=40)\end{array}$ & $\begin{array}{l}\text { The } \\
\text { Other } \\
(n=7)\end{array}$ & & $\begin{array}{c}\geq 2 \\
\text { matched } \\
(n=19)\end{array}$ & $\begin{array}{l}\text { The } \\
\text { other } \\
(n=28)\end{array}$ & & $\begin{array}{c}3 \\
\text { matched } \\
(n=6)\end{array}$ & $\begin{array}{l}\text { The } \\
\text { Other } \\
(n=41)\end{array}$ & \\
\hline Age & & & 0.475 & & & 0.312 & & & 0.312 \\
\hline$<65$ & 19 & 4 & & 11 & 12 & & 4 & 19 & \\
\hline$\geq 65$ & 21 & 3 & & 8 & 16 & & 2 & 22 & \\
\hline Gender & & & 0.229 & & & 0.739 & & & 0.092 \\
\hline Male & 25 & 6 & & 12 & 19 & & 2 & 29 & \\
\hline Female & 15 & 1 & & 7 & 9 & & 4 & 12 & \\
\hline Tumor location & & & 0.197 & & & 0.229 & & & 0.021 \\
\hline Head & 32 & 4 & & 13 & 23 & & 2 & 34 & \\
\hline Body - Tail & 8 & 3 & & 6 & 5 & & 4 & 7 & \\
\hline UICC T classification & & & 0.477 & & & 0.769 & & & 0.092 \\
\hline $\mathrm{T} 3$ & 27 & 4 & & 13 & 18 & & 2 & 29 & \\
\hline $\mathrm{T} 4$ & 13 & 3 & & 6 & 10 & & 4 & 12 & \\
\hline Major vessel resection & & & 0.164 & & & 0.634 & & & 0.378 \\
\hline Yes & 30 & 7 & & 15 & 22 & & 4 & 33 & \\
\hline No & 10 & 0 & & 4 & 6 & & 2 & 8 & \\
\hline Surgical margin & & & 0.512 & & & 0.536 & & & 0.568 \\
\hline R0 & 36 & 7 & & 17 & 26 & & 6 & 37 & \\
\hline $\mathrm{R} 1$ & 4 & 0 & & 2 & 2 & & 0 & 4 & \\
\hline Pathological differentiation & & & 0.276 & & & 0.400 & & & 0.643 \\
\hline Well & 5 & 2 & & 2 & 5 & & 1 & 6 & \\
\hline Others & 35 & 5 & & 17 & 23 & & 5 & 35 & \\
\hline Lymph node metastasis & & & 0.475 & & & 0.172 & & & 0.104 \\
\hline Negative & 21 & 3 & & 12 & 12 & & 5 & 19 & \\
\hline Positive & 19 & 4 & & 7 & 16 & & 1 & 22 & \\
\hline
\end{tabular}

Table VI. Correlation between clinical efficacy of NACRT and expression of biomarkers.

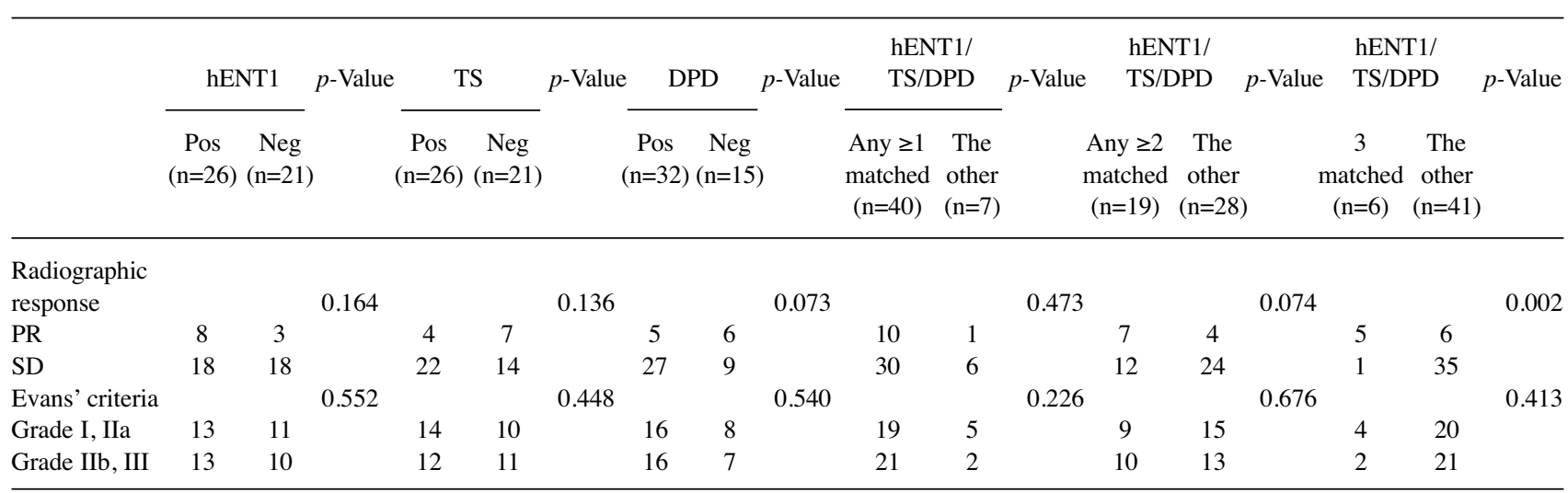

Pos: positive, Neg: negative.

OS of 25 months in patients with BRPC who underwent resection after NACRT at our institute.

A study (29) suggested that hENT1 is a potential biomarker for long-term outcome of GEM-based chemotherapy for pancreatic cancer. The hENT1 expression affected therapeutic outcome of GEM-based NACRT (30). Other clinical studies $(16,31)$ of pancreatic cancer have also revealed that low DPD expression predicts efficacy and prognosis of fluoropyrimidinetreated patients. In addition, TS expression was reported to be a poor prognostic factor for pancreatic cancer patients treated 
Table VII. Univariate and multivariate survival analysis of prognostic factors.

\begin{tabular}{|c|c|c|c|c|c|c|c|c|}
\hline & $\mathrm{N}$ & $\begin{array}{c}\text { DFS } \\
3 \text { year } \\
\text { rate }(\%)\end{array}$ & $p$-Value & $\begin{array}{c}\text { OS } \\
3 \text { year } \\
\text { rate }(\%)\end{array}$ & $p$-Value & HR & $95 \% \mathrm{CI}$ & $p$-Value \\
\hline Age & & & 0.641 & & 0.648 & & & \\
\hline$<65$ & 23 & 34 & & 31 & & & & \\
\hline$\geq 65$ & 24 & 30 & & 38 & & & & \\
\hline Gender & & & 0.734 & & 0.930 & & & \\
\hline Male & 31 & 33 & & 30 & & & & \\
\hline Female & 16 & 28 & & 45 & & & & \\
\hline Tumor location & & & 0.747 & & 0.028 & 2.539 & $0.861-7.489$ & 0.091 \\
\hline Head & 36 & 35 & & 25 & & & & \\
\hline Body - Tail & 11 & 32 & & 59 & & & & \\
\hline Major vessel resection & & & 0.843 & & 0.709 & & & \\
\hline Yes & 37 & 33 & & 36 & & & & \\
\hline No & 10 & 30 & & 27 & & & & \\
\hline Surgical margin & & & 0.832 & & 0.438 & & & \\
\hline R0 & 43 & 33 & & 36 & & & & \\
\hline $\mathrm{R} 1$ & 4 & 25 & & 25 & & & & \\
\hline Lymph node metastasis & & & 0.243 & & 0.134 & & & \\
\hline Negative & 24 & 40 & & 53 & & & & \\
\hline Positive & 23 & 24 & & 17 & & & & \\
\hline Pathological differentiation & & & 0.283 & & 0.630 & & & \\
\hline Well & 7 & 0 & & 0 & & & & \\
\hline Others & 40 & 38 & & 38 & & & & \\
\hline Evan's criteria & & & 0.396 & & 0.190 & & & \\
\hline Grade IIIa & 24 & 38 & & 42 & & & & \\
\hline Grade IIb III & 23 & 28 & & 25 & & & & \\
\hline Adjuvand Chemotherapy & & & 0.301 & & 0.250 & & & \\
\hline Yes & 35 & 33 & & 34 & & & & \\
\hline No & 12 & 32 & & 30 & & & & \\
\hline hENT1 & & & 0.565 & & 0.224 & & & \\
\hline Positive & 26 & 32 & & 37 & & & & \\
\hline Negative & 21 & 30 & & 30 & & & & \\
\hline TS & & & 0.090 & & 0.013 & & & \\
\hline Positive & 26 & 15 & & 10 & & & & \\
\hline Negative & 21 & 48 & & 65 & & & & \\
\hline DPD & & & 0.524 & & 0.035 & & & \\
\hline Positive & 32 & 30 & & 22 & & & & \\
\hline Negative & 15 & 38 & & 59 & & & & \\
\hline \multicolumn{9}{|l|}{ hENT1/TS/DPD } \\
\hline Positive/Negative/Negat & & & & & & & & \\
\hline Any $\geq 1$ matched & 40 & 36 & 0.209 & 37 & 0.339 & & & \\
\hline the other & 7 & 14 & & 17 & & & & \\
\hline Any $\geq 2$ matched & 19 & 52 & 0.056 & 70 & 0.002 & 3.403 & $1.335-8.675$ & 0.010 \\
\hline the other & 28 & 17 & & 10 & & & & \\
\hline 3 matched & 6 & 44 & 0.421 & 100 & 0.005 & & & \\
\hline the other & 41 & 31 & & 23 & & & & \\
\hline
\end{tabular}

with 5-FU-based adjuvant therapy (16). Thus, hENT1, TS, and DPD are candidate biomarkers for GEM concomitant with fluoropyrimidine treatments. In our study, combination analysis of these biomarkers appeared useful for patient selection.

Unexpectedly, expression profiles in FNA specimens were not predictive of outcomes in the patients. Specimens from endoscopic ultrasound-guided FNA may have been too small to reflect the characteristics of the whole tumor. However, the positive predictive values of FNA specimens were relatively high at 71,80 , and $69 \%$, respectively. Yamada et al. reported that pretreatment hENT1 expressions in FNA specimens were concordant with those in the resected specimen after GEM-based chemoradiotherapy (32). Further studies are required to confirm the importance of enzyme expression in FNA specimens. 
a

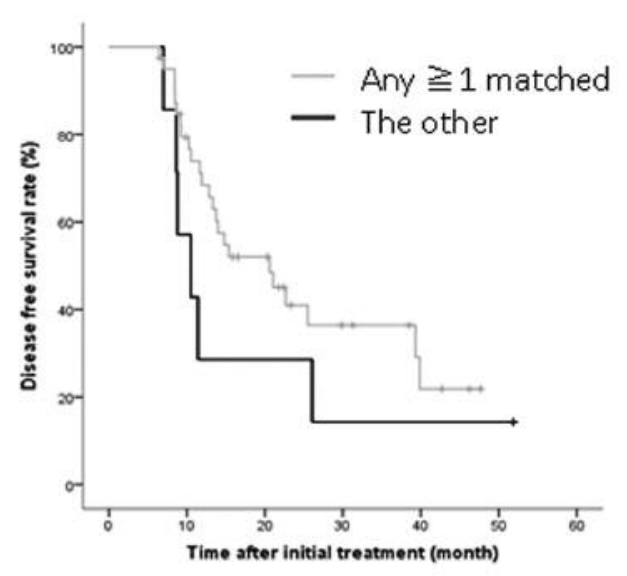

C

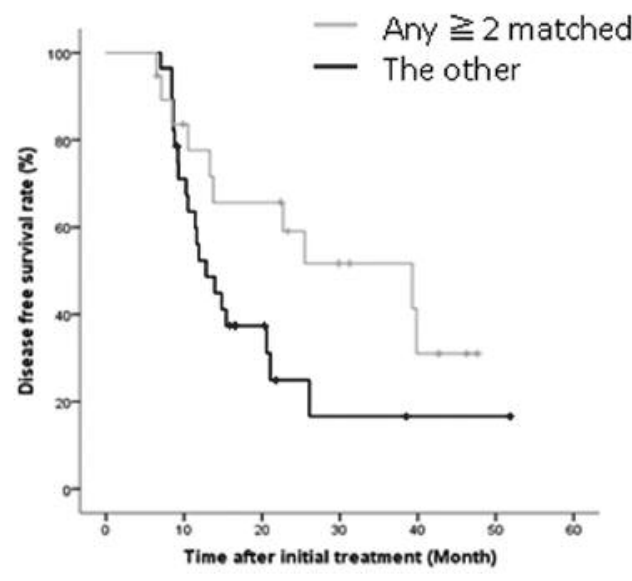

e

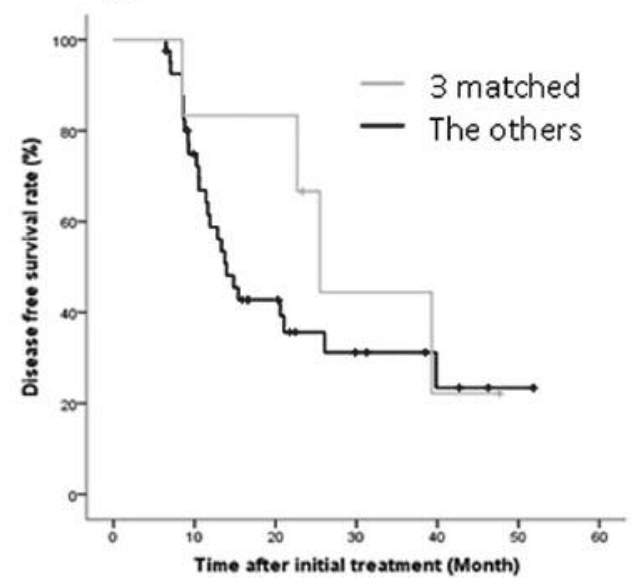

b

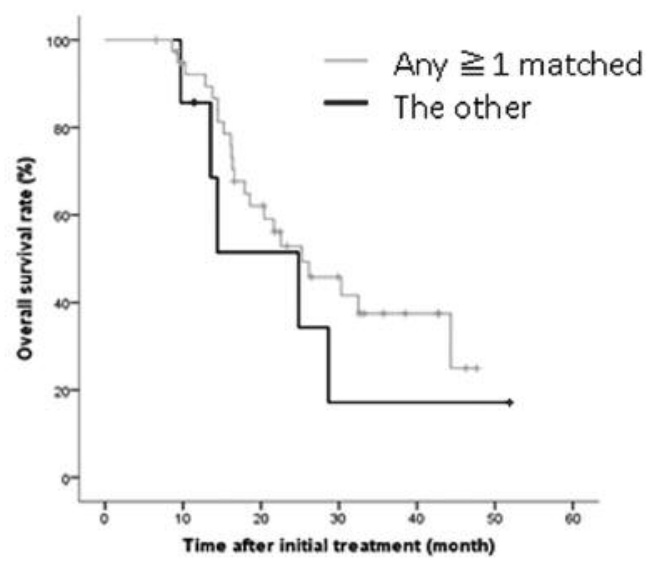

d
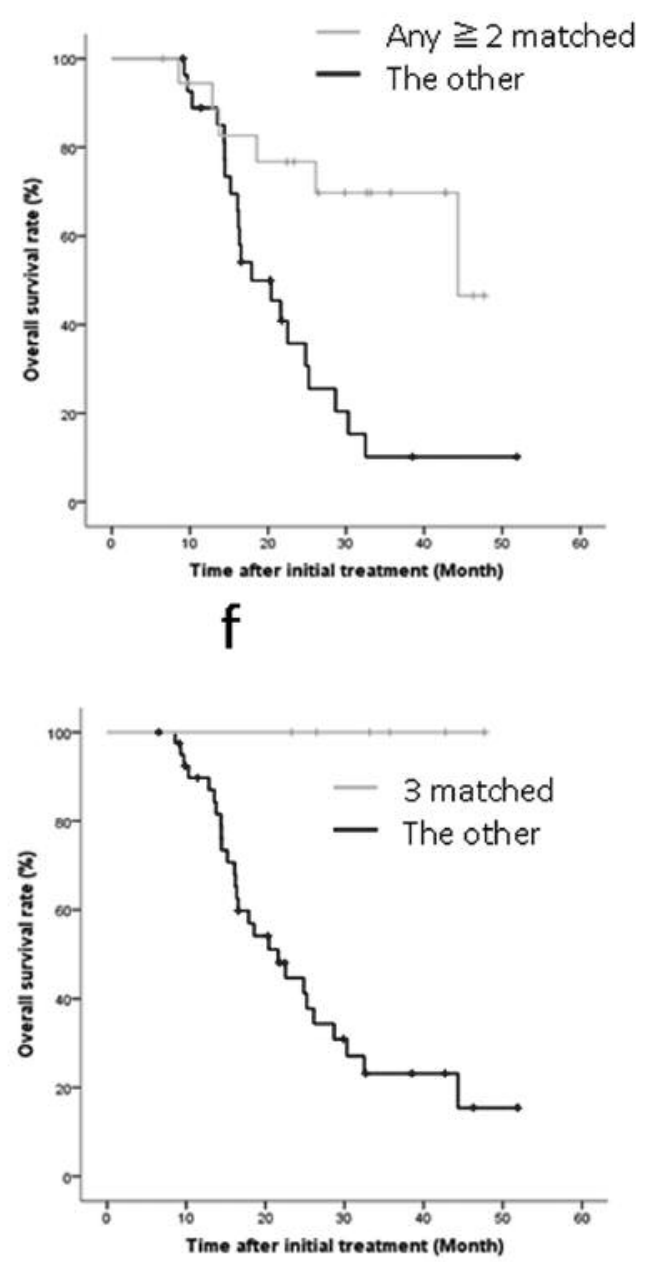

Figure 2. Correlation between combined expression analysis and both of DFS and OS. Patients with more than two favorable factors tended to have longer DFS (c) $p=0.056)$ and had significantly longer $O S(d) p=0.002)$ than patients in other groups. Patients with three favorable factors had significantly longer $O S(f)(p=0.005)$ than patients in other groups. 
S-1 treatments induced an increase of hENT1 mRNA levels and GEM uptake in pancreatic cancer xenografts (33). Treatment with GEM increased hENT1 expression and reduced 5-FU sensitivity in human pancreatic cancer cell lines (34). Thus, in the present study, NACRT using GEM plus S-1 may also have caused changes in the expression of these factors in FNA specimen ie pretreatment tumor cells.

Recent studies indicate a relationship between hENT1 expression and NACRT outcomes in patients with pancreatic cancer. Murata et al. (30) reported positive correlation between hENT1 expression and improved outcome. In contrast, another report described that hENT1 expression did not predict long-term outcomes in patients with pancreatic cancer who underwent NACRT with GEM (35). This discrepancy may reflect the use of GEM as an adjuvant chemotherapy in the former study and the contrasting use of continuous liver perfusions of 5-FU as adjuvant chemotherapy in the latter study. In the present study, adjuvant chemotherapy comprised GEM plus S-1 for 57\%, GEM alone for 9\%, and S-1 alone for $9 \%$ of patients, and $25 \%$ of patients did not undergo any adjuvant chemotherapy. This heterogeneity may have influenced the relationship between hENT1, TS, and DPD expression and long-term survival. TS and DPD were more reliable prognostic factors than hENT1 expression. According to this finding, it is widely accepted that 5-FU metabolism-associated enzymes affect the efficacy of 5-FUbased chemoradiotherapy $(36,37)$. In view of the present results, patients who lack favorable factors may consider adjuvant chemotherapy using drugs other than GEM or S-1, such as modified FOLFIRINOX or GEM and nab-paclitaxel.

Previous reports described independent associations of positive lymph node metastasis and surgical margin status with longer OS in patients with pancreatic cancer who were treated with NACRT $(27,30)$. In the present study, the presence of more than two favorable factors (more than two from hENT1 positive, TS negative, and DPD negative) was selected as an independent favorable prognostic factor of OS for patients with pancreatic cancer who underwent NACRT with GEM plus S-1.

This study had limitations, such as its retrospective nature and a small number of participants. Thus, further prospective validation with a larger patient cohort is required to confirm the results of the present study.

In summary, combined analysis of hENT1, TS amd DPD in resected specimens may predict therapeutic efficacy of NACRT with GEM plus S-1 in patients with BRPC. Further studies are required to determine the efficacy of these biomarkers in FNA specimens. Adjuvant chemotherapy with drugs other than GEM or S-1 should be considered for patients in whom any three factors are absent.

\section{Conflicts of Interest}

The Authors report no potential conflict of interest regarding this study.

\section{Acknowledgements}

The Authors thank Dr. Masashi Momiyama and Ms. Harumi Sakurada for technical assistance

\section{References}

1 Siegel R, Miller KD and Jemal A: Cancer statistics, 2015. CA Cancer J Clin 65: 5-29, 2015.

2 Wray CJ, Ahmad SA, Matthews JB and Lowy AM: Surgery for pancreatic cancer: recent controversies and current practice. Gastroenterology 128: 1626-241, 2005.

3 Wagner M, Redaelli C, Lietz M, Seiler CA, Friess H and Büchler MW: Curative resection is the single most important factor determining outcome in patients with pancreatic adenocarcinoma. Br J Surg 91: 586-594, 2004.

4 Neoptolemos JP, Stocken DD, Friess H, Bassi C, Dunn JA, Hickey H, Beger H, Fernandez-Cruz L, Dervenis C, Lacaine F, Falconi M, Pederzoli P, Pap A, Spooner D, Kerr DJ, Büchler MW and European Study Group for Pancreatic Cancer: A randomized trial of chemoradiotherapy and chemotherapy after resection of pancreatic cancer. N Engl J Med 350: 1200-1210, 2004.

5 Neoptolemos JP, Stocken DD, Friess H, Bassi C, Dunn JA, Hickey H, Beger H, Fernandez-Cruz L, Dervenis C, Lacaine F, Falconi M, Pederzoli P, Pap A, Spooner D, Kerr DJ, Büchler MW and European Study Group for Pancreatic Cancer: Adjuvant chemotherapy with fluorouracil plus folinic acid $v s$. gemcitabine following pancreatic cancer resection: a randomized controlled trial. JAMA 304: 1073-1081, 2010.

6 Oettle H, Post S, Neuhaus P, Gellert K, Langrehr J, Ridwelski K, Schramm H, Fahlke J, Zuelke C, Burkart C, Gutberlet K, Kettner E, Schmalenberg H, Weigang-Koehler K, Bechstein WO, Niedergethmann M, Schmidt-Wolf I, Roll L, Doerken B and Riess $\mathrm{H}$ : Adjuvant chemotherapy with gemcitabine vs. observation in patients undergoing curative-intent resection of pancreatic cancer: a randomized controlled trial. JAMA 297: 267-277, 2007.

7 Evans DB, Rich TA, Byrd DR, Cleary KR, Connelly JH, Levin B, Charnsangavej C, Fenoglio CJ and Ames FC: Preoperative chemoradiation and pancreaticoduodenectomy for adenocarcinoma of the pancreas. Arch Surg 127: 1335-1339, 1992.

8 Katz MH, Pisters PW, Evans DB, Sun CC, Lee JE, Fleming JB, Vauthey JN, Abdalla EK, Crane CH, Wolff RA, Varadhachary GR and Hwang RF: Borderline resectable pancreatic cancer: the importance of this emerging stage of disease. J Am Coll Surg 206: 833-848, 2008 .

9 Kato H, Usui M, Isaji S, Nagakawa T, Wada K, Unno M, Nakao A, Miyakawa S and Ohta T: Clinical features and treatment outcome of borderline resectable pancreatic head/body cancer: a multi-institutional survey by the Japanese Society of Pancreatic Surgery. J Hepatobiliary Pancreat Sci 20: 601-610, 2013.

10 García-Manteiga J, Molina-Arcas M, Casado FJ, Mazo A and Pastor-Anglada M: Nucleoside transporter profiles in human pancreatic cancer cells: role of hCNT1 in 2',2'-difluorodeoxycytidine- induced cytotoxicity. Clin Cancer Res 9: 5000-5008, 2003.

11 Mackey JR, Mani RS, Selner M, Mowles D, Young JD, Belt JA, Crawford CR and Cass CE: Functional nucleoside transporters are required for gemcitabine influx and manifestation of toxicity in cancer cell lines. Cancer Res 58: 4349-4357, 1998. 
12 Mori R, Ishikawa T, Ichikawa Y, Taniguchi K, Matsuyama R, Ueda M, Fujii Y, Endo I, Togo S, Danenberg PV and Shimada $\mathrm{H}$ : Human equilibrative nucleoside transporter 1 is associated with the chemosensitivity of gemcitabine in human pancreatic adenocarcinoma and biliary tract carcinoma cells. Oncol Rep 17: 1201-1205, 2007.

13 Farrell JJ, Elsaleh H, Garcia M, Lai R, Ammar A, Regine WF, Abrams R, Benson AB, Macdonald J, Cass CE, Dicker AP and Mackey JR: Human equilibrative nucleoside transporter 1 levels predict response to gemcitabine in patients with pancreatic cancer. Gastroenterology 136: 187-195, 2009.

14 Ueno H, Ioka T, Ikeda M, Ohkawa S, Yanagimoto H, Boku N, Fukutomi A, Sugimori K, Baba H, Yamao K, Shimamura T, Sho M, Kitano M, Cheng AL, Mizumoto K, Chen JS, Furuse J, Funakoshi A, Hatori T, Yamaguchi T, Egawa S, Sato A, Ohashi Y, Okusaka T and Tanaka M: Randomized phase III study of gemcitabine plus S-1, S-1 alone, or gemcitabine alone in patients with locally advanced and metastatic pancreatic cancer in Japan and Taiwan: GEST study. J Clin Oncol 31: 1640$1648,2013$.

$15 \mathrm{Hu}$ YC, Komorowski RA, Graewin S, Hostetter G, Kallioniemi OP, Pitt HA and Ahrendt SA: Thymidylate synthase expression predicts the response to 5-fluorouracil-based adjuvant therapy in pancreatic cancer. Clin Cancer Res 9: 4165-4171, 2003.

16 Nakayama S, Takeda S, Kawase Y, Inoue S, Kaneko T and Nakao A: Clinical significance of dihydropyrimidine dehydrogenase in adjuvant 5-fluorouracil liver perfusion chemotherapy for pancreatic cancer. Ann Surg 240: 840-844, 2004.

17 Matsubara J, Nishina T, Yamada Y, Moriwaki T, Shimoda T, Kajiwara T, Nakajima TE, Kato K, Hamaguchi T, Shimada Y, Okayama Y, Oka T and Shirao K: Impacts of excision repair cross-complementing gene 1 (ERCC1), dihydropyrimidine dehydrogenase, and epidermal growth factor receptor on the outcomes of patients with advanced gastric cancer. Br J Cancer 98: 832-839, 2008.

18 Ishido K1, Azuma M, Koizumi W, Takeuchi A, Sakuramoto S, Watanabe $M$ and Okayasu I: Evaluation of prognostic factors for the response to $\mathrm{S}-1$ in patients with stage II or III advanced gastric cancer who underwent gastrectomy. Pharmacogenet Genomics 19: 955-964, 2009.

19 National Comprehensive Cancer Network (NCCN). Clinical practice guidelines in oncology. Pancreatic Adenocarcinoma. Version 1. 2013.

20 Eisenhauer EA, Therasse P, Bogaerts J, Schwartz LH, Sargent D, Ford R, Dancey J, Arbuck S, Gwyther S, Mooney M, Rubinstein L, Shankar L, Dodd L, Kaplan R, Lacombe D and Verweij $\mathrm{J}$ : New response evaluation criteria in solid tumors: revised RECIST guideline (version 1.1). Eur J Cancer 45: 228247,2009

21 (UICC) IUAC. TMN classification of malignant tumors. 7th ed. New York: Wiley-Liss, 2010.

22 Johnston PG, Fisher ER, Rockette HE, Fisher B, Wolmark N, Drake JC, Chabner BA, and Allegra CJ: The role of thymidylate synthase expression in prognosis and outcome of adjuvant chemotherapy in patients with rectal cancer. J Clin Oncol 12: 2640-2647, 1994.

23 Kamoshida S1, Shiogama K, Shimomura R, Inada K, Sakurai Y, Ochiai M, Matuoka H, Maeda K and Tsutsumi Y: Immunohistochemical demonstration of fluoropyrimidine-metabolizing enzymes in various types of cancer. Oncol Rep 14: 1223-1230, 2005.
24 Burris HA 3rd, Moore MJ, Andersen J, Green MR, Rothenberg ML, Modiano MR, Cripps MC, Portenoy RK, Storniolo AM, Tarassoff P, Nelson R, Dorr FA, Stephens CD and Von Hoff DD: Improvements in survival and clinical benefit with gemcitabine as first-line therapy for patients with advanced pancreas cancer: a randomized trial. J Clin Oncol 15: 2403-2413, 1997.

25 Regine WF, Winter KA, Abrams RA, Safran H, Hoffman JP, Konski A, Benson AB, Macdonald JS, Kudrimoti MR, Fromm ML, Haddock MG, Schaefer P, Willett CG and Rich TA: Fluorouracil vs. gemcitabine chemotherapy before and after fluorouracil-based chemoradiation following resection of pancreatic adenocarcinoma: a randomized controlled trial. JAMA 299: 1019-1026, 2008.

26 Oettle H, Post S, Neuhaus P, Gellert K, Langrehr J, Ridwelski K, Schramm H, Fahlke J, Zuelke C, Burkart C, Gutberlet K, Kettner $\mathrm{E}$, Schmalenberg $\mathrm{H}$, Weigang-Koehler $\mathrm{K}$, Bechstein WO, Niedergethmann M, Schmidt-Wolf I, Roll L, Doerken B and Riess $\mathrm{H}$ : Adjuvant chemotherapy with gemcitabine $v s$. observation in patients undergoing curative-intent resection of pancreatic cancer: a randomized controlled trial. JAMA 297: 267-277, 2007.

27 Evans DB, Varadhachary GR, Crane CH, Sun CC, Lee JE, Pisters PW, Vauthey JN, Wang H, Cleary KR, Staerkel GA, Charnsangavej C, Lano EA, Ho L, Lenzi R, Abbruzzese JL and Wolff RA: Preoperative gemcitabine-based chemoradiation for patients with resectable adenocarcinoma of the pancreatic head. J Clin Oncol 26: 3496-3502, 2008.

28 Motoi F, Ishida K, Fujishima F, Ottomo S, Oikawa M, Okada T, Shimamura H, Takemura S, Ono F, Akada M, Nakagawa K, Katayose Y, Egawa S and Unno M: Neoadjuvant Chemotherapy with Gemcitabine and S-1 for Resectable and Borderline Pancreatic Ductal Adenocarcinoma: Results from a Prospective Multi-institutional Phase 2 Trial. Ann Surg Oncol 20: 3794$3801,2013$.

29 Giovannetti E1, Del Tacca M, Mey V, Funel N, Nannizzi S, Ricci S, Orlandini C, Boggi U, Campani D, Del Chiaro M, Iannopollo M, Bevilacqua G, Mosca $\mathrm{F}$ and Danesi R: Transcription analysis of human equilibrative nucleoside transporter-1 predicts survival in pancreas cancer patients treated with gemcitabine. Cancer Res 66: 3928-3935, 2006.

30 Murata Y, Hamada T, Kishiwada M, Ohsawa I, Mizuno S, Usui M, Sakurai H, Tabata M, Ii N, Inoue H, Shiraishi T and Isaji S: Human equilibrative nucleoside transporter 1 expression is a strong independent prognostic factor in UICC T3-T4 pancreatic cancer patients treated with preoperative gemcitabine-based chemoradiotherapy. J Hepatobiliary Pancreat Sci 19: 413-425, 2012.

31 Kondo N, Murakami Y, Uemura K, Sudo T, Hashimoto Y, Nakashima A, Ohge $\mathrm{H}$ and Sueda T. Prognostic impact of dihydropyrimidine dehydrogenase expression on pancreatic adenocarcinoma patients treated with S-1-based adjuvant chemotherapy after surgical resection. J Surg Oncol 104: 146$154,2011$.

32 Yamada R, Mizuno S, Uchida K, Yoneda M, Kanayama K, Inoue H, Murata Y, Kuriyama N, Kishiwada M, Usui M, Ii N, Tsuboi J, Tano S, Hamada Y, Tanaka K, Horiki N, Ogura T, Shiraishi T, Takei Y, Katayama N and Isaji S: Human Equilibrative Nucleoside Transporter 1 Expression in Endoscopic Ultrasonography-Guided Fine-Needle Aspiration Biopsy Samples Is a Strong Predictor of Clinical Response and Survival in the Patients With Pancreatic Ductal Adenocarcinoma Undergoing Gemcitabine-Based Chemoradiotherapy. Pancreas 45: 761-771, 2016. 
33 Nakahira S, Nakamori S, Tsujie M, Takeda S, Sugimoto K, Takahashi Y, Okami J, Marubashi S, Miyamoto A, Takeda Y, Nagano H, Dono K, Umeshita K, Sakon M and Monden M: Pretreatment with S-1, an oral derivative of 5-fluorouracil, enhances gemcitabine effects in pancreatic cancer xenografts. Anticancer Res 28: 179-186, 2008.

34 Rauchwerger DR, Firby PS, Hedley DW and Moore MJ: Equilibrative-sensitive nucleoside transporter and its role in gemcitabine sensitivity. Cancer Res 60: 6075-6079, 2000.

35 Kawada N, Uehara H, Katayama K, Nakamura S, Takahashi H, Ohigashi $\mathrm{H}$, Ishikawa $\mathrm{O}$, Nagata $\mathrm{S}$ and Tomita $\mathrm{Y}$ : Human equilibrative nucleoside transporter 1 level does not predict prognosis in pancreatic cancer patients treated with neoadjuvant chemoradiation including gemcitabine. J Hepatobiliary Pancreat Sci 19: 717-722, 2012.
36 Liersch T, Langer C, Ghadimi BM, Kulle B, Aust DE, Baretton GB, Schwabe W, Häusler P, Becker Ha and Jakob C: Lymph node status and TS gene expression are prognostic markers in stage II/III rectal cancer after neoadjuvant fluorouracil-based chemoradiotherapy, J. Clin. Oncol 24: 4062-4068, 2006.

37 Tanaka K, Saigusa S, Toiyama Y, Koike Y, Okugawa Y, Yokoe T, Inoue Y, Kobayashi M, Miki C and Kusunoki M: TS and DPD mRNA levels on formalin-fixed paraffin-embedded specimens as predictors for distant recurrence of rectal cancer treated with preoperative chemoradiotherapy. J Surg Oncol 105: 529-534, 2012 .

Received March 4, 2017

Revised March 31, 2017

Accepted April 4, 2017 